\title{
A comparison of dosimetric variance for external-beam partial breast irradiation using three-dimensional and four-dimensional computed tomography
}

\author{
This article was published in the following Dove Press journal: \\ OncoTargets and Therapy \\ 31 March 2016 \\ Number of times this article has been viewed
}

\section{Bing Guo ${ }^{1,2}$ \\ Jian-Bin $\mathrm{Li}^{2}$ \\ Wei Wang ${ }^{2}$ \\ Min $X u^{2}$ \\ Yan-Kang $\mathrm{Li}^{2,3}$ \\ Tong-Hai Liu²}

'School of Medicine and Life Sciences, University of Jinan-Shandong Academy of Medical Sciences, Jinan, Shandong Province, People's Republic of China; 2Department of Radiation Oncology, Shandong Cancer Hospital \& Institute, Jinan, Shandong Province, People's Republic of China; ${ }^{3}$ School of Medicine, Shandong University, Jinan, Shandong Province, People's Republic of China
Correspondence: Jian-Bin Li

Department of Radiation Oncology,

Shandong Cancer Hospital \& Institute,

Number 440 Jiyan Road, Jinan 250I I7,

Shandong Province, People's Republic of

China

Tel +8653167626131

Fax +86 53I 6762 6130

Email lijianbin@msn.com
Purpose: To investigate the potential dosimetric benefits from four-dimensional computed tomography (4DCT) compared with three-dimensional computed tomography (3DCT) in radiotherapy treatment planning for external-beam partial breast irradiation (EB-PBI).

Patients and methods: 3DCT and 4DCT scan sets were acquired for 20 patients who underwent EB-PBI. The volume of the tumor bed (TB) was determined based on seroma or surgical clips on 3DCT images (defined as $\mathrm{TB}_{3 \mathrm{D}}$ ) and the end inhalation (EI) and end exhalation (EE) phases of 4DCT images (defined as $\mathrm{TB}_{\mathrm{EI}}$ and $\mathrm{TB}_{\mathrm{EE}}$, respectively). The clinical target volume (CTV) consisted of the TB plus a $1.0 \mathrm{~cm}$ margin. The planning target volume (PTV) was the CTV plus $0.5 \mathrm{~cm}$ (defined as $\mathrm{PTV}_{3 \mathrm{D}}, \mathrm{PTV}_{\mathrm{EI}}$, and $\mathrm{PTV}_{\mathrm{EE}}$ ). For each patient, a conventional 3D conformal plan (3D-CRT) was generated (defined as EB-PBI ${ }_{3 \mathrm{D}}, \mathrm{EB}_{\mathrm{PBI}} \mathrm{EI}_{\mathrm{E}}$, and $\mathrm{EB}-\mathrm{PBI} \mathrm{EE}_{\mathrm{EE}}$ ).

Results: The $\mathrm{PTV}_{3 \mathrm{D}}, \mathrm{PTV}_{\mathrm{EI}}$, and $\mathrm{PTV}_{\mathrm{EE}}$ were similar $(P=0.549)$, but the PTV coverage of EB-PBI ${ }_{3 \mathrm{D}}$ was significantly less than that of $\mathrm{EB}-\mathrm{PBI}_{\mathrm{EI}}$ or $\mathrm{EB}-\mathrm{PBI}_{\mathrm{EE}}(P=0.001$ and $P=0.025$, respectively). There were no significant differences in the homogeneity or conformity indexes between the three treatment plans $\left(P=0.125\right.$ and $P=0.536$, respectively). The EB-PBI ${ }_{3 \mathrm{D}}$ plan resulted in the largest organs at risk dose.

Conclusion: There was a significant benefit for patients when using 3D-CRT based on 4DCT for EB-PBI with regard to reducing nontarget organ exposure. Respiratory motion did not affect the dosimetric distribution during free breathing, but might result in poor dose coverage when the PTV is determined using 3DCT.

Keywords: breast cancer, external-beam partial breast irradiation, three-dimensional computed tomography, four-dimensional computed tomography, dosimetric parameters

\section{Introduction}

Breast-conserving therapy, which involves a wide local excision followed by radiotherapy to the whole breast, is accepted as the standard treatment for early-stage breast carcinoma. ${ }^{1}$ The efficacy of breast-conserving therapy for early-stage breast carcinoma was established in randomized trials., ${ }^{2,3}$ Regardless of whether whole-breast irradiation is delivered, the vast majority of ipsilateral breast cancers recur in the tumor bed (TB), and remote recurrences are rare. ${ }^{4}$ However, administration of a larger irradiation dose per fraction might help to maintain tumor control and desirable cosmetic results; therefore, external-beam partial breast irradiation (EB-PBI) may be a better treatment choice than whole-breast irradiation. Hyperfractionated accelerated radiation therapy facilitates the administration of 1-10 fractions delivered over 5 days. EB-PBI irradiates 
the surgical cavity and surrounding breast tissue; therefore, target volume definition, setup error correction, and measures to limit respiration-induced target movements are critical considerations for EB-PBI planning. ${ }^{1,5}$

Computed tomography (CT) simulation and accurate determination of target margins are prerequisites for successful oncology radiotherapy. Respiration-induced motion during irradiation is a major geometric uncertainty that may affect treatment accuracy. Therefore, dosimetric variation across the target resulting from intrafraction motion could be a significant concern. ${ }^{6}$ Temporal images acquired during conventional axial three-dimensional computed tomography (3DCT) may include limited respiratory motion details for determining the target margin, but it has been difficult to evaluate how much motion information the 3DCT provides. ${ }^{7}$ In contrast, the fourdimensional computed tomography (4DCT) is a reliable and effective tool for assessing tumor motion. ${ }^{8}$ 4DCT enables the correlation of $\mathrm{CT}$ data acquisition with respiratory cycles, facilitating the acquisition of a series of 3DCT data sets over a patient's breathing cycle to provide visualization of tumor motion on an individual patient basis. ${ }^{9}$

For EB-PBI planning, recent studies have investigated delineating the TB using seroma or surgical clips based on 3DCT and 4DCT during free breathing. ${ }^{10-12}$ However, the variability of the specified dosimetric parameters for the TB and organs at risk (OARs) between 3DCT and 4DCT planning has not been established. To investigate the impact of these modalities for EB-PBI treatment planning, we analyzed and compared the treatment plans based on 3DCT images, and the end inhalation (EI) and end exhalation (EE) phases of 4DCT images.

\section{Methods and materials}

\section{Patients}

Twenty patients who underwent wide local excision of the breast followed by superficial closure (ten left-sided and ten right-sided lesions) for EB-PBI between June 2009 and November 2013 were included in this study. All enrolled patients had a seroma clarity score of $3-5,{ }^{13}$ and $\geq 5$ round surgical clips ( $2 \mathrm{~mm}$ in diameter) were used to mark the boundaries of the lumpectomy cavity. The surgical clips were fixed to the cranial, caudal, medial, lateral, and dorsal walls of the surgical cavity. None of the patients had chronic lung diseases, and all of them exhibited normal ventilation function. This study was approved by the Institutional Review Board of the Shandong Tumour Hospital Ethics Committee, and written informed consent was obtained from all patients.

\section{DCT and 4DCT data acquisition}

Patients were immobilized in the supine position on a breast board using an arm support (with both arms above the head to expose the breast adequately). The 3DCT and 4DCT data sets were acquired using a 16-slice CT scanner (Philips Brilliance Bores CT, Best, the Netherlands) during free breathing.

The 3DCT scans, in which 12 contiguous slices with a thickness of $2 \mathrm{~mm}$ were produced per gantry rotation (1 second with a 1.8-second interval between rotations), were acquired in sequence. The 4DCT scanning was performed in the helical mode with the scanning pitch set at 0.09-0.15. The respiratory signals were sent to the scanner to label each 4DCT image with a time tag. GE Advantage 4D software (General Electric Healthcare, Waukesha, WI, USA) was used to sort the reconstructed 4DCT images into ten respiratory phases based on these tags, with $0 \%$ corresponding to EI and $50 \%$ corresponding to EE. The constructed 4DCT image sets were subsequently transferred to the Eclipse treatment planning system (Eclipse ${ }^{\mathrm{TM}}$ 8.6; Varian Medical Systems, Palo Alto, CA, USA) for structure delineation.

\section{Treatment planning and dosimetric evaluation}

All TBs were delineated by the same radiation oncologist using the same window and level setting for 3DCT and 4DCT images. The TB was delineated based on both the clips and the seroma (defined as $\mathrm{TB}_{3 \mathrm{D}}, \mathrm{TB}_{\mathrm{El}}$, and $\mathrm{TB}_{\mathrm{EE}}$ ). The clinical target volume (CTV) consisted of the TB plus a $1.0 \mathrm{~cm}$ margin, and the planning target volume (PTV) consisted of the CTV plus a $0.5 \mathrm{~cm}$ margin (defined as $\mathrm{PTV}_{3 \mathrm{D}}, \mathrm{PTV}_{\mathrm{El}}$, and $\mathrm{PTV}_{\mathrm{EE}}$, respectively). Both the CTV and PTV were limited to $5 \mathrm{~mm}$ from the skin surface and lung-chest wall interface. ${ }^{14}$ The TB volume and PTV were recorded for each patient. The ipsilateral normal breast was delineated based on the 3DCT and 4DCT images. In addition, the ipsilateral lungs and heart (left-sided lesions) were contoured based on the 3DCT and 4DCT images.

In all cases, three-dimensional conformal radiotherapy (3D-CRT) with $6 \mathrm{MV}$ photons in a 4-field noncoplanar beam arrangement was employed. The treatment plan based on the 4DCT EI phase images was copied and applied to the EE phase and 3DCT images (defined as EB-PBI ${ }_{\mathrm{El}}, \mathrm{EB}^{\mathrm{PBB}} \mathrm{EE}_{\mathrm{EE}}$, and $\mathrm{EB}_{-\mathrm{PBI}_{3 \mathrm{D}}}$, respectively) with the same gantry angles, collimator angles, primary field size, and monitor units delivered per beam. The prescription dose was $34 \mathrm{~Gy}$, which was given in 10 fractions (3.4 Gy per fraction administered twice daily) to the PTV using $6 \mathrm{MV}$ photon beams, which was defined as the $90 \%$ isodose line. The criterion of the 3D-CRT EB-PBI 
treatment plan was to ensure that at least $95 \%$ of the PTV received the prescribed dose.

Dose distribution was calculated separately for each treatment plan, and dose-volume histogram parameters for the PTV, ipsilateral normal breast, ipsilateral lungs, and heart were calculated for each plan in all patients. The mean dose $\left(D_{\text {mean }}\right)$, homogeneity index (HI), and conformal index (CI) were evaluated for the PTV. HI was defined as follows:

$$
\mathrm{HI}=\frac{D_{2}-D_{98}}{\text { prescribed dose }}
$$

where $D_{2}$ and $D_{98}$ represent the doses covering $2 \%$ and $98 \%$ of the PTV, respectively. ${ }^{15,16} \mathrm{CI}$ was defined as follows:

$$
\mathrm{CI}=\frac{\begin{array}{c}
\text { Ref .isodose volume } \\
\text { of the PTV }
\end{array}}{\text { PTV }} \times \frac{\begin{array}{c}
\text { Ref.isodose volume } \\
\text { of the PTV }
\end{array}}{\text { Ref.isodose volume }}
$$

where ref.isodose-volume of the PTV $\left(\mathrm{PTV}_{\text {ref }}\right)$ represents the PTV that is covered by the prescribed dose, and ref. isodose-volume $\left(V_{\text {ref }}\right)$ is the volume enclosed by the prescribed isodose. ${ }^{15,16}$ The ipsilateral lungs and heart were evaluated using the $D_{\text {mean }}$ and the volumes that received $\geq 5,10$, or 20 Gy $\left(V_{5}, V_{10}\right.$, and $V_{20}$, respectively). The ipsilateral normal breast was evaluated using the $D_{\text {mean }}$ and the volumes that received $\geq 20$ or 30 Gy ( $V_{20}$ and $V_{30}$, respectively).

\section{Statistical analyses}

SPSS 19.0 software (IBM Corporation, Armonk, NY, USA) was used for statistical analysis. Because the variables did not follow a normal distribution, the data were summarized using medians and ranges. Friedman tests were performed to establish the variability of each dosimetric parameter, TB volume, and PTV between each treatment plan. All significant effects were investigated post hoc using Wilcoxon signedranks tests. For all tests, a $P$-value $<0.05$ was considered statistically significant.

\section{Results \\ Target volumes}

The $\mathrm{TB}_{3 \mathrm{D}}$ volume was significantly greater than that of $\mathrm{TB}_{\mathrm{EI}}$ and $\mathrm{TB}_{\mathrm{EE}}$ volumes $(P=0.002$ each); however, there was no significant difference between the $\mathrm{TB}_{\mathrm{EI}}$ and $\mathrm{TB}_{\mathrm{EE}}$ volumes $(P=0.737)$. The volume variability between $\mathrm{PTV}_{3 \mathrm{D}}, \mathrm{PTV}_{\mathrm{EI}}$, and $\mathrm{PTV}_{\mathrm{EE}}$ was not statistically significant $(P=0.549)$. For the ipsilateral breast volume, there was no difference between the EI, EE, and 3DCT images $(P=0.086)$ (Table 1).

\section{PTV parameters}

The HI and CI of the PTVs are listed in Table 2. The HI and CI did not differ significantly between each plan $(P=0.125$ and $P=0.536$, respectively). The PTV coverage of EB-PBI ${ }_{3 \mathrm{D}}$ was significantly less than that of $\mathrm{EB}-\mathrm{PBI}_{\mathrm{EI}}$ or $\mathrm{EB}-\mathrm{PBI}{ }_{\mathrm{EE}}$ ( $P=0.001$ and $P=0.025$, respectively). The PTV coverage for the EB-PBI ${ }_{\mathrm{EI}}$ and $\mathrm{EB}-\mathrm{PBI}_{\mathrm{EE}}$ treatment plans did not differ significantly $(P=0.668)$. Similar mean dose variability was observed between the $\mathrm{PTV}_{3 \mathrm{D}}, \mathrm{PTV}_{\mathrm{EI}}$, and $\mathrm{PTV}_{\mathrm{EE}}(P=0.132)$ (Table 2).

\section{Dose to organs at risk}

Table 3 shows the variation of the specified dose and volume parameters for the ipsilateral normal breast, ipsilateral lungs, and heart for each plan. A comparison of these parameters for treatment planning based on the imaging is detailed in Table 4.

For the ipsilateral normal breast, the mean dose, $V_{20}$, and $V_{30}$ obtained using the EB-PBI $\mathrm{EI}_{\mathrm{EI}}$ and $\mathrm{EB}-\mathrm{PBI}_{\mathrm{EE}}$ treatment plans were all significantly lower than those using the EB-PBI treatment plan $(P<0.05)$. There was no significant difference in the dose received between the EB-PBI ${ }_{\mathrm{EI}}$ and $\mathrm{EB}-\mathrm{PBI} \mathrm{EE}_{\mathrm{E}}$ treatment plans $(P>0.05)$ (Table 4$)$.

Similarly, for the ipsilateral lungs, the mean dose, $V_{5}, V_{10}$, and $V_{20}$ acquired using the EB-PBI ${ }_{\mathrm{EI}}$ and $\mathrm{EB}-\mathrm{PBI}_{\mathrm{EE}}$ treatment plans were significantly lower than those using the EB-PBI ${ }_{3 \mathrm{D}}$ treatment plan $(P<0.05)$. There was no significant difference between the doses received using the $\mathrm{EB}-\mathrm{PBI}_{\mathrm{EI}}$ and $\mathrm{EB}-\mathrm{PBI} \mathrm{EE}_{\mathrm{E}}$ treatment plans $(P>0.05)$ (Table 4$)$.

There was a significant difference between the treatment

\begin{tabular}{|c|c|c|c|}
\hline Parameter & 3DCT & EI & EE \\
\hline $\mathrm{TB}$ & $20.99(|I .4|-90.35)$ & 19.28 (II.33-88.42) & | 8.78 (I I.48-88.|7) \\
\hline PTV & I 60.63 (9I.60-297.45) & I54.43 (I0I.49-298.57) & | 52.44 (99.40-28|.80) \\
\hline Ipsilateral breast & $517.24(306.76-1,089.20)$ & $534.56(302.04-1,1$ I 6.09) & $531.40(302.05-1,106.60)$ \\
\hline
\end{tabular}
plans with regard to the mean dose, $V_{5}$, or $V_{10}$ received by the

Table I Target volume based on the three different phases $\left(\mathrm{cm}^{3}\right)$

Note: Data format is median (minimum - maximum) as calculated by SPSS 19.0 software.

Abbreviations: 3DCT, three-dimensional computed tomography; El, end inhalation; EE, end exhalation; TB, tumor bed; PTV, planning target volume. 
Table 2 Planning target volume parameters for the three treatment plans

\begin{tabular}{|c|c|c|c|c|c|}
\hline Parameter & EB-PBI ${ }_{3 \mathrm{D}}$ & EB-PBI $_{\mathrm{EI}}$ & EB-PBI $\left.\right|_{E E}$ & $\mathbf{z}$ & $P$-value \\
\hline $\mathrm{HI}$ & $0.13(0.10-0.20)$ & $0.13(0.10-0.21)$ & $0.13(0.10-0.22)$ & 4.154 & 0.125 \\
\hline $\mathrm{Cl}$ & $0.68(0.56-0.74)$ & $0.69(0.53-0.75)$ & $0.68(0.52-0.77)$ & 1.246 & 0.536 \\
\hline$V_{90 \%}$ & 96.85 (94.32-99.48) & 97.51 (95-99.45) & 97.03 (94.34-99.5I) & 7.500 & 0.024 \\
\hline$D_{\text {mean }}$ & $36.20(35.63-36.82)$ & $36.20(34.49-36.82)$ & $36.22(35.62-36.74)$ & 4.055 & 0.132 \\
\hline
\end{tabular}

Notes: Data format is median (minimum - maximum) as calculated by SPSS 19.0 software. P-value was calculated by Friedman test.

Abbreviations: $\mathrm{EB}^{\mathrm{PBB}}{ }_{3 \mathrm{D}}$, treatment plan established based on $3 \mathrm{DCT}$; $\mathrm{EB}-\mathrm{PBI} \mathrm{El}_{\mathrm{I}}$, treatment plan established based on end inhalation; $\mathrm{EB}-\mathrm{PBI} \mathrm{EE}$, treatment plan established based on end exhalation; 3DCT, three-dimensional computed tomography; $\mathrm{HI}$, homogeneity index; $\mathrm{Cl}$, conformity index; $\mathrm{V}_{90 \%}$, percentage of the planning target volume for evaluation receiving the prescribed dose (\%); $D_{\text {mean }}$, mean dose (Gy).

heart $(P=0.004,0.008,0.018$, respectively) (Table 3). The specified doses for the heart were significantly lower using the 4DCT images $(P<0.05)$. The EB-PBI ${ }_{\mathrm{EI}}$ and $\mathrm{EB}-\mathrm{PBI} \mathrm{EE}_{\mathrm{E}}$ treatment plans showed similar doses $(P>0.05)$ (Table 4$)$.

\section{Discussion}

Respiratory motion and cardiac contractions could lead to tumor displacement and deformity, so delineation of the TB is a critical component of radiotherapy for thoracic tumors, especially for EB-PBI. In addition, determining the accuracy of treatment delivery for each fraction and assessing the need for heterogeneity correction proposes further challenges for treatment planning. In recent years, 4D image-based motion capture has improved treatment planning for patients with breast cancer. ${ }^{10,12,16}$

For EB-PBI, the gross tumor volume is the lumpectomy cavity or the seroma volume, and the CTV is generally defined as the contouring of the seroma or clips within the lumpectomy cavity, expanded by some margin, usually $1.5 \mathrm{~cm}$. An additional $0.5-1.0 \mathrm{~cm}$ margin is usually added to obtain the PTV. ${ }^{17,18}$ We used this approach in the previous study; however, this approach has been questioned because the universal expansion of the lumpectomy cavity sometimes results in a PTV that is too large to be accommodated in patients with small breasts. ${ }^{19}$ Compared with female Western patients, female Chinese patients with breast cancer typically have a smaller breast volume, earlier disease onset, and smaller body stature. Therefore, we ensured that the "total" TB-to-PTV margin, which was employed to accommodate organ motion and setup error $(1.5 \mathrm{~cm})$, was sufficient to accommodate the observed uncertainty for accurate delivery.

Volume variance, caused by residual motion artifacts during imaging, ${ }^{20}$ is considered a geometrical uncertainty that could affect EB-PBI treatment. 4DCT is widely used to estimate volumetric variations in target volumes throughout the respiratory cycle. In this study, there was a significant difference between the EI, EE, and 3DCT images in terms of determining the TB volumes; $\mathrm{TB}_{3 \mathrm{D}}$ had the greatest volume. However, the PTV ${ }_{3 \mathrm{D}}, \mathrm{PTV}_{\mathrm{EI}}$, and $\mathrm{PTV}_{\mathrm{EE}}$ did not differ significantly, most likely because of exclusion of the PTV expansion within the lungs and the part outside of the skin surface. Previous studies have suggested that the intraobserver and interobserver variability in breast target volume delineation decreases according to a standard contouring protocol. ${ }^{12,21}$

Table 3 Dosimetric evaluation for the three treatment plans (median, \%)

\begin{tabular}{|c|c|c|c|c|c|}
\hline Parameter & EB-PBI $_{3 D}$ & EB-PBI ${ }_{E I}$ & EB-PBI ${ }_{E E}$ & $\mathbf{z}$ & $P$-value \\
\hline \multicolumn{6}{|c|}{ Ipsilateral normal breast } \\
\hline$D_{\text {mean }}$ & | 3.85 (8.69-19.79) & $13.72(8.62-19.55)$ & | 3.67 (8.9|-|8.82) & 10.81 & 0.004 \\
\hline$V_{20}^{\text {mean }}$ & $28.83(19.72-46.78)$ & $28.66(19.35-45.79)$ & $28.18(19.15-43.56)$ & 10.90 & 0.004 \\
\hline $\begin{array}{l}V_{30} \\
\text { Ipsilateral lung }\end{array}$ & I7.8I (9.59-30.94) & $17.02(9.47-30.17)$ & $17.04(9.08-31.29)$ & 11.20 & 0.004 \\
\hline$D_{\text {mean }}$ & $2.15(0.63-3.97)$ & $2.17(0.72-3.91)$ & $2.18(0.66-4.01)$ & 6.10 & 0.047 \\
\hline$V_{5}$ & II.48 (I.53-19.50) & $10.69(1.96-18.98)$ & $10.66(1.79-19.75)$ & 6.40 & $0.04 I$ \\
\hline$V_{10}$ & $6.73(0.70-13.31)$ & $6.48(0.75-12.95)$ & $6.64(0.75-13.63)$ & 8.39 & 0.015 \\
\hline $\begin{array}{l}V_{20} \\
\text { Heart }\end{array}$ & $1.72(0.11-5.58)$ & $1.83(0.14-5.33)$ & $1.73(0.10-5.55)$ & 7.00 & 0.030 \\
\hline$D_{\text {mean }}$ & $0.76(0.26-3.46)$ & $0.56(0.27-3.18)$ & $0.60(0.21-3.43)$ & 11.13 & 0.004 \\
\hline$V_{5}$ & $3.05(0.00-20.70)$ & $1.78(0.00-18.98)$ & $2.04(0.00-17.21)$ & 9.74 & 0.008 \\
\hline$V_{10}$ & $1.10(0.00-14.85)$ & $0.44(0.00-13.40)$ & $0.54(0.00-11.86)$ & 8.00 & 0.018 \\
\hline
\end{tabular}

Notes: Data format is median (minimum - maximum) as calculated by SPSS 19.0 software. $P$-value was calculated by Friedman test.

Abbreviations: $\mathrm{EB}^{\mathrm{PBB}}{ }_{3 \mathrm{D}}$, treatment plan established based on $3 \mathrm{DCT}$; $\mathrm{EB}-\mathrm{PBI} \mathrm{E}_{\mathrm{E}}$, treatment plan established based on end inhalation; $\mathrm{EB}-\mathrm{PBI} \mathrm{EE}_{\mathrm{E}}$, treatment plan established based on end exhalation; 3DCT, three-dimensional computed tomography; $D_{\text {mean }}$, mean dose (Gy); $V_{5}$, volume that received $\geq 5$ Gy; $V_{10}$, volume that received $\geq 10 \mathrm{~Gy}$; $V_{20}$, volume that received $\geq 20 \mathrm{~Gy} ; V_{30}$, volume that received $\geq 30 \mathrm{~Gy}$. 
Table $4 \mathrm{~A}$ comparison of the parameters for treatment planning based on the three different CT images

\begin{tabular}{|c|c|c|c|c|c|c|}
\hline \multirow[t]{2}{*}{ Parameter } & \multicolumn{2}{|l|}{ EI-EE } & \multicolumn{2}{|c|}{ EI-3DCT } & \multicolumn{2}{|c|}{ EE-3DCT } \\
\hline & $z$ & $P$-value & $\mathbf{z}$ & $P$-value & $z$ & $P$-value \\
\hline \multicolumn{7}{|c|}{ Ipsilateral normal breast } \\
\hline$D_{\text {mean }}$ & -1.326 & 0.185 & -2.390 & 0.017 & -2.354 & 0.019 \\
\hline$V_{20}$ & -0.635 & 0.526 & -3.062 & 0.002 & -2.277 & 0.023 \\
\hline$V_{30}$ & -1.008 & 0.313 & -2.837 & 0.005 & -2.837 & 0.005 \\
\hline \multicolumn{7}{|c|}{ Ipsilateral lungs } \\
\hline$D_{\text {mean }}$ & -0.342 & 0.732 & -2.499 & 0.014 & -2.297 & 0.022 \\
\hline$V_{5}$ & -1.232 & 0.218 & -3.211 & 0.001 & -2.259 & 0.024 \\
\hline$V_{10}$ & -1.248 & 0.212 & -3.285 & 0.001 & -2.173 & 0.030 \\
\hline$v_{20}$ & -0.101 & 0.920 & -1.993 & 0.046 & -2.634 & 0.008 \\
\hline \multicolumn{7}{|l|}{ Heart } \\
\hline$D_{\text {mean }}$ & -0.971 & 0.322 & -2.547 & 0.011 & -2.807 & 0.005 \\
\hline$V_{5}$ & -0.507 & 0.612 & -2.521 & 0.012 & -2.100 & 0.036 \\
\hline$V_{10}$ & -0.676 & 0.499 & -2.366 & 0.018 & -2.197 & 0.028 \\
\hline
\end{tabular}

Notes: Data format is median (minimum - maximum) as calculated by SPSS 19.0 software. $P$-value was calculated by Wilcoxon-signed-ranks test.

Abbreviations: 3DCT, three-dimensional computed tomography; El, end inhalation; $E E$, end exhalation; $D$, mean dose $(G y) ; V_{5}$, volume that received $\geq 5$ $\mathrm{Gy} ; V_{10}$, volume that received $\geq 10 \mathrm{~Gy} ; V_{20}$, volume that received $\geq 20 \mathrm{~Gy} ; V_{30}$, volume that received $\geq 30 \mathrm{~Gy}$.

Therefore, in our study, all delineations were conducted by a single radiation oncologist using specific guidelines to decrease geometrical uncertainties. Breast volume did not show a significant change throughout free breathing, which indicated that the breast deformation during normal respiration could be ignored for EB-PBI.

A valuable feature of $4 \mathrm{DCT}$ is that respiratory motion can be assessed so that population-based or site-specific tumor motion information can be acquired to guide the expansion of the TB based on 3DCT. Because 3DCT does not encompass detailed motion information, our premise was that the $\mathrm{PTV}_{3 \mathrm{D}}$ coverage would not be greater than that of the PTV coverage for a single breathing phase; otherwise any expansion would result in more amount of normal tissue being unnecessarily irradiated. Our data supported this hypothesis. The PTV coverage of EB-PBI ${ }_{3 \mathrm{D}}$ was significantly less than that of EB-PBI ${ }_{\mathrm{EI}}$ or EB-PBI ${ }_{\mathrm{EE}}$. We showed that the radiation dose required for $4 \mathrm{D}$ image acquisitions could be effectively managed. Therefore, caution should be used when the individual $\mathrm{PTV}_{3 \mathrm{D}}$ is used in treatment planning, especially for patients with an irregular breathing pattern.

Knopf et $\mathrm{al}^{22}$ reported that using multiple scanning beam directions improved dose homogeneity in patients with liver conditions. In addition, Kumar et $\mathrm{al}^{23}$ suggested that low doses were delivered to the target and OARs, and a better $\mathrm{HI}$ and $\mathrm{CI}$ were obtained for EB-PBI treatment. In the present study, dosimetric analysis demonstrated that low dose EB-PBI using clips and seroma was technically feasible for
OARs. In addition, we observed no significant differences in HI or CI between the three treatment plans, and the PTV mean dose for each plan was similar, which suggested that respiratory motion did not have a remarkable influence on the dosimetric distribution during free breathing. Using 4DCT images, Wang et $\mathrm{al}^{6}$ demonstrated that no significant difference between the EE and EI phases planning for either $\mathrm{CI}$ or $\mathrm{HI}$ was found. However, statistical significance was found between 3D and EI, and between 3D and EE. The differences between the results in our study and Wang et al's investigation ${ }^{6}$ might be due to the differences in radiotherapy technique. We treated the TB with 3D-CRT for EB-PBI, while Wang et $\mathrm{al}^{6}$ used conventional SMLC-intensity-modulated radiotherapy planning for whole breast. In addition to the differences in radiotherapy technique, other factors which may contribute to respiration-associated dose deterioration include large, pendulous breasts, lumpectomy bed near the edge of the treatment fields that more likely move out of the fields, and superficial tumors that may be anatomically more susceptible to movement.

Breathing rate influences the effective dose because a reduced pitch factor is required in patients with a lower breathing rate. ${ }^{24}$ However Qi et al ${ }^{25}$ reported that, for a series of 18 patients, during normal breathing, the dosimetric impact of respiratory motion was clinically insignificant, with the exception of the internal mammary nodes. In the present study, the specified doses for the OARs were not significantly different between the EB-PBI ${ }_{\mathrm{EI}}$ and $\mathrm{EB}-\mathrm{PBI}_{\mathrm{EE}}$ treatment plans, whereas those for the EB-PBI ${ }_{3 \mathrm{D}}$ treatment plan were significantly higher. Liao et $\mathrm{al}^{8}$ investigated 3D-CRT EB-PBI based on 4DCT in patients with early-stage breast cancer. They found that the 4DCT-planned prescribed dose levels for the ipsilateral breast, ipsilateral lungs, and heart were significantly lower compared with 3DCT-planned dose levels, which was congruent with the findings of the present study. Furthermore, using 4DCT images, Rodríguez-Romero and Castro-Tejero ${ }^{26}$ found that organ position was altered during breathing, which resulted in higher volume discrepancies when breath depth, length, or frequency increased. From these results, we determined that the 4DCT treatment plan was clearly superior to 3DCT treatment plan for the protection of OARs. This provided a reference for using an adaptive radiotherapy technique for EB-PBI. Furthermore, Cover et $\mathrm{al}^{27}$ reported that when the EE phase was reviewed in the sagittal plane, gating would reduce the mean tumor mobility from $6.3 \pm 2.0$ to $1.4 \pm 0.5 \mathrm{~mm}$. In the process of target volume delineation, we also found that the end-exhalation phase was the most stable phase on 4DCT images. Therefore, 
the delineation of the target volume and performing treatment plan based on EE phase may be more reasonable in radiotherapy treatment planning.

However, some limitations and inaccuracies remained in the study. First, our study focused on the dosimetric variance of the TB and OARs for EB-PBI based on 3DCT and 4DCT images without knowledge of the pathological information on the real tumor and the follow-ups. Another issue was that the target volume delineation errors were not considered. However, this uncertainty might affect the accuracy of the dose distribution and dose homogeneity for the treatment of the breast cancer. Therefore, all targets and OARs should be consistently delineated by one radiotherapist using uniform criteria to decrease interobserver variance.

\section{Conclusion}

Compared with 3DCT, we could benefit more from the use of 4DCT-based planning of 3D-CRT for EB-PBI in patients with breast cancer. Respiratory motion did not have a remarkable influence on dose distribution during free breathing, but could result in suboptimal dose coverage of the PTV when 3DCT is used for planning. Furthermore, 4DCT-based planning could significantly reduce the exposure of nontarget organs to irradiation. While we do not suggest that 4DCT should act as a replacement for $3 \mathrm{DCT}$, we do suggest that the benefits of 4DCT planning would be most apparent in patients with an irregular breathing pattern.

\section{Acknowledgments}

This manuscript was edited for English language by Elsevier.

\section{Disclosure}

The authors report no conflicts of interest in this work.

\section{References}

1. Clarke M, Collins R, Darby S, et al. Effects of radiotherapy and of differences in the extent of surgery for early breast cancer on local recurrence and 15-year survival: an overview of the randomised trials. Lancet Oncol. 2005;366(9503):2087-2106.

2. Litiere S, Werutsky G, Fentiman IS, et al. Breast conserving therapy versus mastectomy for stage I-II breast cancer: 20 year follow-up of the EORTC 10801 phase 3 randomised trial. Lancet Oncol. 2012;13(4): 412-419.

3. Blichert-Toft M, Nielsen M, During M, et al. Long-term results of breast conserving surgery vs. mastectomy for early stage invasive breast cancer: 20-year follow-up of the Danish randomized DBCG-82TM protocol. Acta Oncol. 2008;47(4):672-681.

4. Kuerer HM, Julian TB, Strom EA, et al. Accelerated partial breast irradiation after conservative surgery for breast cancer. Ann Surg. 2004;239(3): $338-351$.
5. Offersen BV, Overgaard M, Kroman N, Overgaard J. Accelerated partial breast irradiation as part of breast conserving therapy of early breast carcinoma: a systematic review. Radiother Oncol. 2009;90(1): $1-13$.

6. Wang W, Li JB, Hu HG, et al. Evaluation of dosimetric variance in whole breast forward-planned intensity-modulated radiotherapy based on 4DCT and 3DCT. J Radiat Res. 2013;54(4):755-761.

7. Li F, Li J, Zhang Y, et al. Geometrical differences in gross target volumes between 3DCT and 4DCT imaging in radiotherapy for nonsmall-cell lung cancer. $J$ Radiat Res. 2013;54(5):950-956.

8. Liao ZW, Guan XX, Li FY, et al. Accelerated partial breast irradiation: use of four-dimensional CT for target localization and assessment of intrafractional motion. Oncol Res. 2010;18(10):503-507.

9. Shang DP, Liu CX, Yin Y. A comparison of the different 3D CT scanning modes on the GTV delineation for the solitary pulmonary lesion. Radiat Oncol. 2014;9:211.

10. Wang S, Li J, Wang W, et al. A study on the displacements of the clips in surgical cavity for external-beam partial breast irradiation after breast-conserving surgery based on 4DCT. $J$ Radiat Res. 2012;53(3): 433-438.

11. Dzhugashvili M, Tournay E, Pichenot C, et al. 3D-conformal accelerated partial breast irradiation treatment planning: the value of surgical clips in the delineation of the lumpectomy cavity. Radiat Oncol. 2009;4:70.

12. Guo B, Li JB, Wang W, et al. Interobserver variability in the delineation of the tumour bed using seroma and surgical clips based on 4DCT scan for external-beam partial breast irradiation. Radiat Oncol. 2015;10:66.

13. Petersen RP, Truong PT, Kader HA, et al. Target volume delineation for partial breast radiotherapy planning: clinical characteristics associated with low interobserver concordance. Int J Radiat Oncol Biol Phys. 2007;69(1):41-48.

14. Lei RY, Leonard CE, Howell KT, et al. External beam accelerated partial breast irradiation yields favorable outcomes in patients with prior breast augmentation. Front Oncol. 2014;4:154.

15. Iqbal K, Isa M, Buzdar SA, Gifford KA, Afzal M. Treatment planning evaluation of sliding window and multiple static segments technique in intensity modulated radiotherapy. Rep Pract Oncol Radiother. 2013; 18(2):101-106.

16. Wang W, Li JB, Hu HG, et al. Correlation between target motion and the dosimetric variance of breast and organ at risk during whole breast radiotherapy using 4DCT. Radiat Oncol. 2013;8:111.

17. Vicini FA, Remouchamps V, Wallace M, et al. Ongoing clinical experience utilizing $3 \mathrm{D}$ conformal external beam radiotherapy to deliver partialbreast irradiation in patients with early-stage breast cancer treated with breast-conserving therapy. Int J Radiat Oncol Biol Phys. 2003;57(5): $1247-1253$.

18. Njeh CF, Saunders MW, Langton CM. Accelerated partial breast irradiation using external beam conformal radiation therapy: a review. Crit Rev Oncol Hematol. 2012;81(1):1-20.

19. Moon SH, Shin KH, Kim TH, et al. Dosimetric comparison of four different external beam partial breast irradiation techniques: threedimensional conformal radiotherapy, intensity-modulated radiotherapy, helical tomotherapy, and proton beam therapy. Radiother Oncol. 2009; 90(1):66-73.

20. Rietzel E, Pan T, Chen GT. Four-dimensional computed tomography: image formation and clinical protocol. Med Phys. 2005;32(4): 874-889.

21. Hurkmans CW, Borger JH, Pieters BR, Russell NS, Jansen E. Variability in target volume delineation on CT scans of the breast. Int $J$ Radiat Oncol Biol Phys. 2001;50(5):1366-1372.

22. Knopf AC, Hong TS, Lomax A. Scanned proton radiotherapy for mobile targets - the effectiveness of re-scanning in the context of different treatment planning approaches and for different motion characteristics. Phys Med Biol. 2011;56(22):7257-7271.

23. Kumar R, Sharma SC, Kapoor R, Singh R, Bhardawaj A. Dosimetric evaluation of $3 \mathrm{D}$ conformal accelerated partial-breast irradiation vs. whole-breast irradiation: a comparative study. Int J Appl Basic Med Res. 2012;2(1):52-57. 
24. Bedi C, Kron T, Willis D, Hubbard P, Milner A, Chua B. Comparison of radiotherapy treatment plans for left-sided breast cancer patients based on three- and four-dimensional computed tomography imaging. Clin Oncol. 2011;23(9):601-607.

25. Qi XS, White J, Rabinovitch R, et al. Respiratory organ motion and dosimetric impact on breast and nodal irradiation. Int J Radiat Oncol Biol Phys. 2010;78(2):609-617.
26. Rodríguez-Romero R, Castro-Tejero P. The influence of respiratory motion on CT image volume definition. Med Phys. 2014;41(4):1-11.

27. Cover KS, Lagerwaard FJ, Senan S: Color intensity projections: a rapid approach for evaluating four-dimensional CT scans in treatment planning. Int J Radiat Oncol Biol Phys. 2006;64(3):954-961.

\section{Publish your work in this journal}

OncoTargets and Therapy is an international, peer-reviewed, open access journal focusing on the pathological basis of all cancers, potential targets for therapy and treatment protocols employed to improve the management of cancer patients. The journal also focuses on the impact of management programs and new therapeutic agents and protocols on

\section{Dovepress}

patient perspectives such as quality of life, adherence and satisfaction. The manuscript management system is completely online and includes a very quick and fair peer-review system, which is all easy to use. Visit http://www.dovepress.com/testimonials.php to read real quotes from published authors.

Submit your manuscript here: http://www.dovepress.com/oncotargets-and-therapy-journal 